J Neurol Neurosurg Psychiatry 2005; 76(Suppl V):v25-v34. doi: 10.1136/jnnp.2005.081885

C ognitive neurology deals mainly with disorders of memory (for example, is the patient's poor memory due to early dementia or to anxiety/depression?) or language (as in stroke). It

should be remembered, however, that other areas of cognition may be selectively impaired. This review will cover disorders of perception and of higher order motor output, both in terms of pathological loss and pathological gain of function.

\title{
PERCEPTION AND ITS DISORDERS
}

A patient must be conscious in order to perceive the world around them. An exploration of consciousness is outside the remit of this article though recently reviewed by others. ${ }^{12}$ The patient must also have the capacity to attend selectively in order to focus on one part of the sensorium. Perceptual processing is then necessary to identify what is being perceived through the various sensory modalities (namely vision, hearing, touch, smell, taste), thus allowing access to semantic knowledge and through this understanding of the environment.

Initially, perceptual information is basic and modality specific, but as it is processed by higher order centres, meaning is ascribed to percepts, and information becomes multi-modal (fig 1). Ultimately, semantic knowledge is accessed using the various sensory streams. For example, if standing in the path of an oncoming train, basic perception will involve visual information, hearing the train coming, and feeling vibration from the ground. These separate streams then come together, accessing relevant semantic knowledge and thus allowing the individual to understand what is taking place. In discussing perception, I shall focus mainly on vision and hearing, as the other three forms of perception are of lesser clinical importance.

Perception is not a passive process, but is modulated by attention. There is feedback from higher order centres down to primary sensory cortex. Similarly, attention influences what is perceived. Attention itself has many levels or subcomponents, including selective, divided, sustained attention, etc. Of relevance to perception is selective attention, a process by which the individual focuses specifically on particular areas of sensory experience, rather than simply passively absorbing all such experience. Patients with subcortical dementia can lose this ability to attend selectively, resulting in increased distractibility due to inability to ignore background extraneous stimuli.

To some extent, we see what we expect to see. For example, if waiting for someone at a crowded venue, we may have several false positive recognitions of strangers because we are primed to expect to see the friend.

\section{VISION}

\section{Normal visual processing}

Visual processing involves signal relay from the retina via the lateral geniculate nucleus to the striate cortex (area V1). In the visual cortex itself, there is initially strong retinotopic localisation, such that striate occipital lesions cause deficits restricted to segments of the visual field. Extrastriate visual cortex is organised more by process than by visual field location-that is, different areas of extrastriate cortex are involved in colour, motion perception, etc. Extrastriate deficits therefore cause deficits in aspects of vision, such as perception of motion, affecting the entire visual field.

Broadly speaking, extrastriate regions group into two streams: the ventral occipitotemporal "what" stream is involved in object recognition, while the occipitoparietal "where" stream is involved in spatial processing. ${ }^{3}$ The ventral stream runs below the calcarine fissure into the medial temporal lobe, while the dorsal stream extends superolaterally from the striate cortex into occipitoparietal and temporoparieto-occipital areas.

Correspondence to:
Dr John D W Greene, Southern General Hospital, Institute of Neurological Sciences, 1345 Govan Road, Glasgow, G51 4TF, UK; john. greene@sgh.scot.nhs.uk

\section{Deficits of visual processing, and how to test for them}

Deficits in visual attention can result in neglect, whereby attention is no longer directed either to self-that is, body part—or to external objects such as food on a plate. 

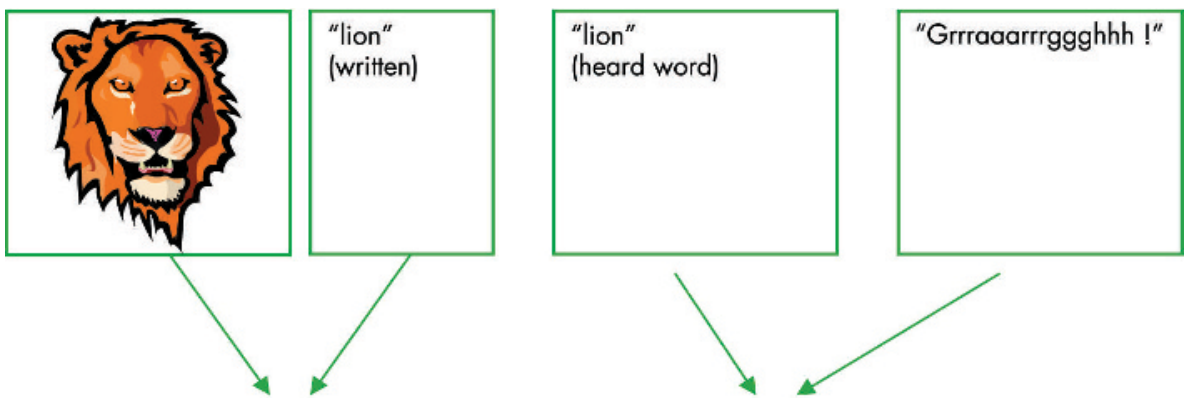

Figure 1 The semantic system illustrating the sensory modalities which can access semantic knowledge.

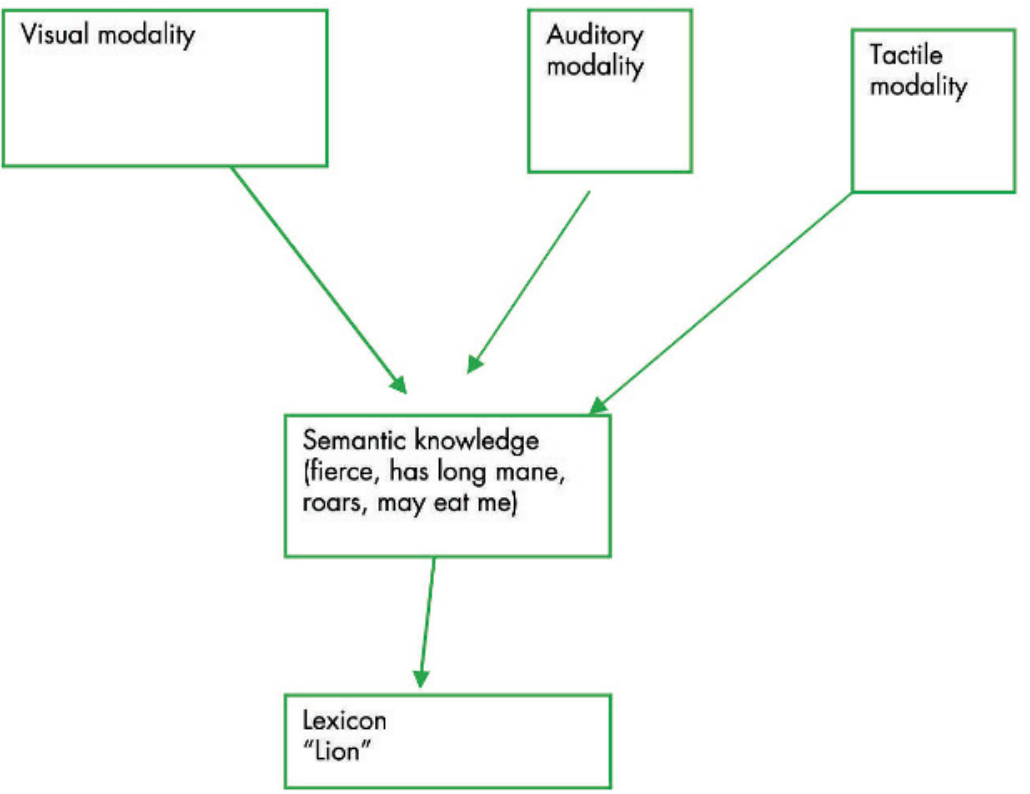

Neglect is almost always for the left hemifield (figs 2 and 3). This is explained by the left hemisphere monitoring the right hemispace, while the right hemisphere monitors both hemispaces. A left hemisphere lesion will still allow the right hemisphere to survey the entire visual field, hence neglect does not occur. By contrast, with a right hemisphere lesion, the left hemisphere monitors only the right hemispace; therefore because of the lack of monitoring the left hemispace, left sided neglect occurs.

Sensory inattention and neglect may be assessed clinically by the examiner moving one, other, or both fingers in right and left hemifields and asking the patient which finger has moved. In visual extinction, the patient will note either finger moving separately, but will only detect the finger in their right hemifield when presented simultaneously. This is due to the ipsilesional stimulus "extinguishing" the contralesional stimulus from awareness. Similarly line bisection tasks can be employed in the clinic (fig 4), as can letter cancellation tasks in a visual array. Drawing a clock face is another useful test (fig 5).

Anosognosia refers to a patient's inability to recognise consciously the presence of somatic dysfunction indicative of a disease process. Patients with left hemiplegia caused by stroke may be entirely unaware of their deficit. Anosognosia tends to occur predominantly, though not exclusively, with non-dominant parietal lesions and is regarded more a disorder of attention than of perception.

Moving next to visual perception itself, clearly retinal, optic nerve pathology or any lesion of the system from the retina via the lateral geniculate nucleus to the primary visual cortex will affect vision. While we are concerned with higher order visual deficits, such confounding conditions must be excluded as part of the general assessment and examination.

Lesions to the ventral or dorsal streams of visual processing can give rise to different clinical deficits. Ventral stream lesions (fig 6) may produce defects such as object agnosia, prosopagnosia, alexia and achromatopsia, while dorsal stream defects (fig 7) include akinetopsia and Balint syndrome. These will be discussed further below.

\section{Disorders of ventral stream Agnosias}

The term agnosia is used to describe a modality specific inability to access semantic knowledge of an object or other stimulus that cannot be attributed to impairment of basic perceptual processes - that is, it relates to a normal percept stripped of its meaning. Agnosias may apply to any sensory modality, but here we shall deal firstly with visual agnosias.

Visual agnosics cannot recognise by sight objects that they would have previously known. They can neither produce unique semantic identifying information nor name the percept. Visual agnosia is sometimes further subdivided into apperceptive visual agnosia, where a deficit in high level perception is implicated (usually widespread, bilateral occipitotemporal infarction), and associative visual agnosia, where high level perception is preserved but the percept is unable to activate semantic identifying information (usually anterior left temporal lobe). The term visual agnosia is best restricted to the inability to access semantic information 


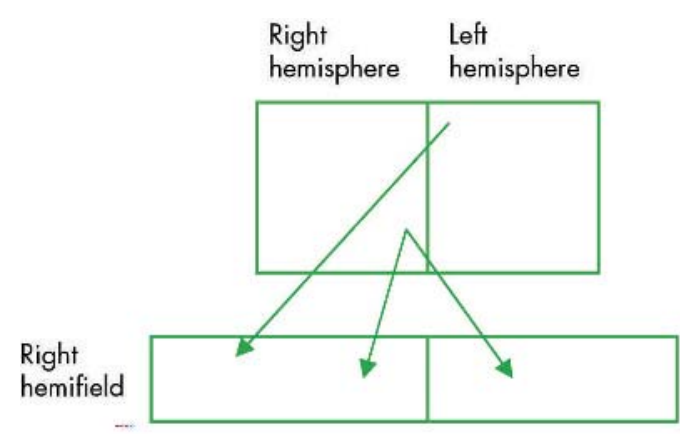

Left hemifield
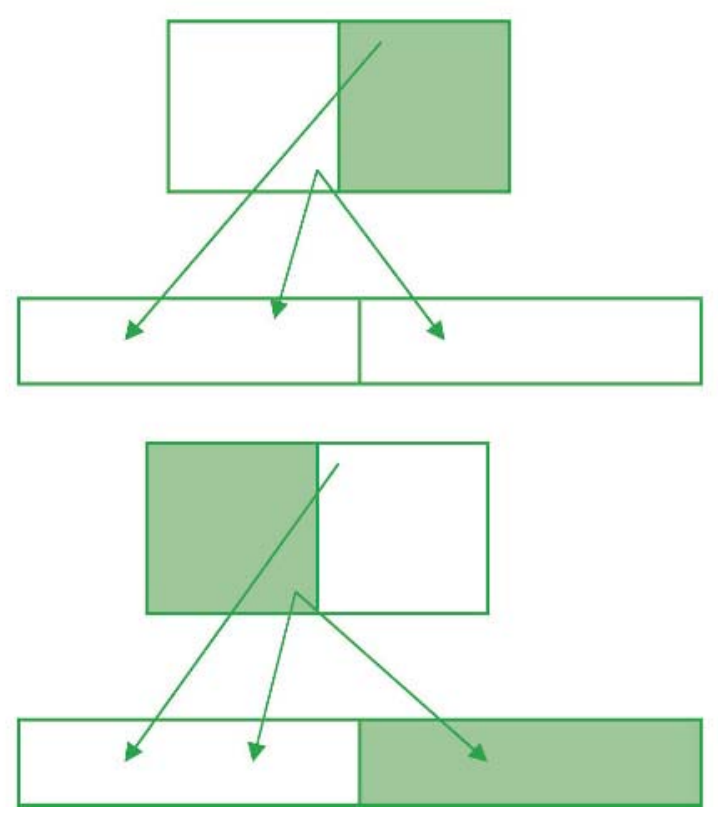

Figure 2 Left hemisphere damage results in no visual field deficit, while right hemisphere damage results in neglect to the left.

through the visual modality only, with retained semantic knowledge accessible through other modalities such as auditory. So called associative visual agnosia often involves a more general loss of semantic knowledge, with no ability to access this via any sensory modality.

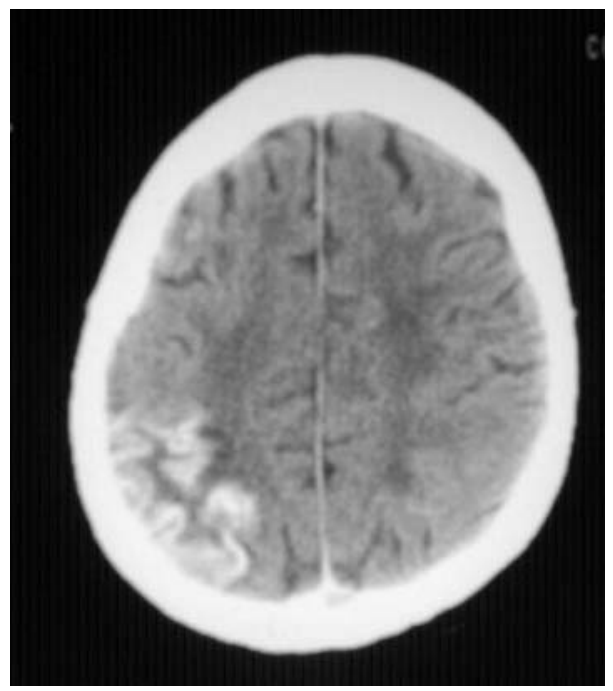

Figure 3 Right gyriform enhancement of right parietal infarct.
Figure 4 Neglect patient does not "see" left half of line, so when asked to put a cross halfway along the line, he puts it halfway along the "seen" line-that is, three quarters of the way along the line.

A generalised visual agnosia may occur in a diffuse hypoxic insult, such as in carbon monoxide poisoning. However, more selective agnosias may also occur. Indeed, deficits may selectively impair ability to identify words or faces (that is, dyslexia and prosopagnosia, respectively), and can occur with focal temporal lesions (fig 8).

\section{Testing for visual agnosia}

Bedside cognitive tests include object naming and ability to provide semantic information about unnamed items. Visuoperceptual function can be tested by asking the patient to draw the object or copy a drawing. The patient can be asked to describe what is seen, and mime its use. If holding the object allows the patient to identify the object where vision has not, this would be in keeping with a visual agnosia rather than a loss of semantic knowledge. (In the latter, identifying information will not be able to be provided regardless of modality of sensory input.)

Bedside testing may be usefully amplified by a formal neuropsychological evaluation. Visuo-perceptual function may be tested by unusual views tests, overlapping line drawings, partially degraded or fragmented images, judgement of line orientation, face analysis, and matching from different angles as well as the Visual Object and Space Perception battery.

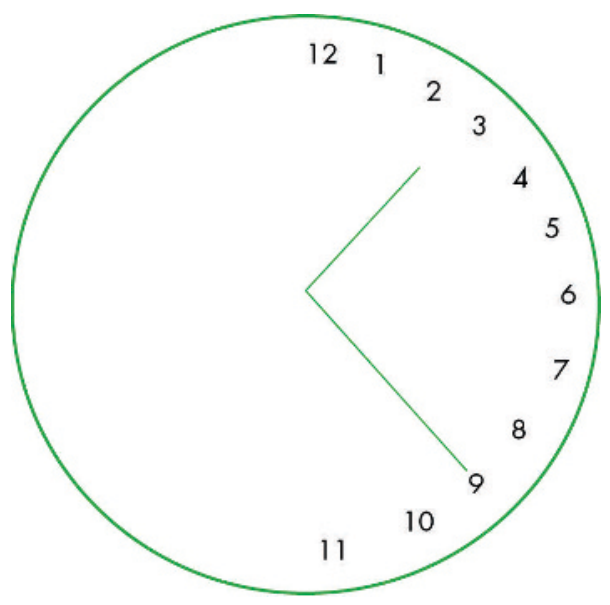

Figure 5 Clockface illustrating neglect. Patient is not able to attend to left hemispace, and puts numbers all on right half of clockface. 


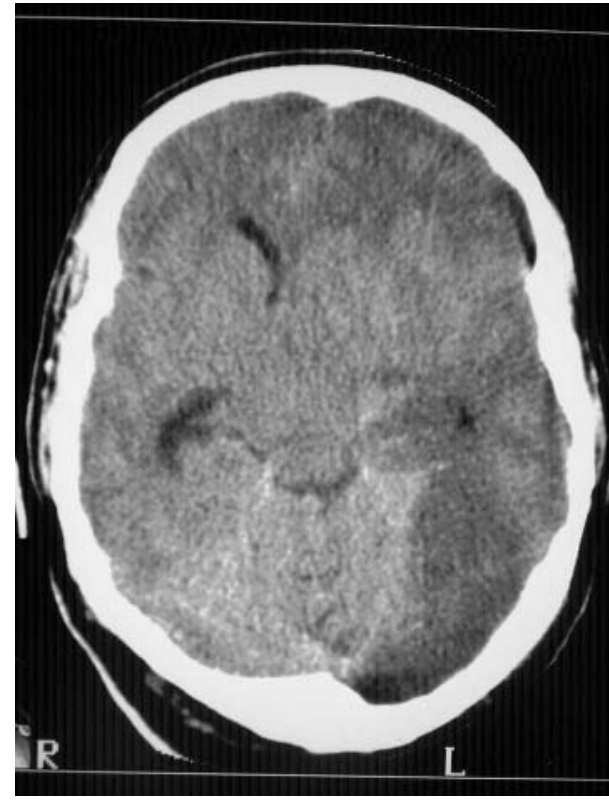

Figure 6 Ventral stream lesion: computed tomographic (CT) scan showing left posterior cerebral artery infarct.

\section{Alexia}

Language is a specialised symbolic representation of the world around us, allowing us to communicate our inner thoughts with others. With regard to perception, language may be accessed through the visual or auditory modality, namely reading or hearing speech respectively.

Reading is a very complex activity. It requires the eye to fixate on the written word, perception, eye movements, and central language to allow understanding of the written word. Deficits in any of these areas can impair the ability to readthat is, alexia. Alexia may be peripheral (where there is difficulty transmitting the visual percept to the intact language centres) or central (due to an impaired language

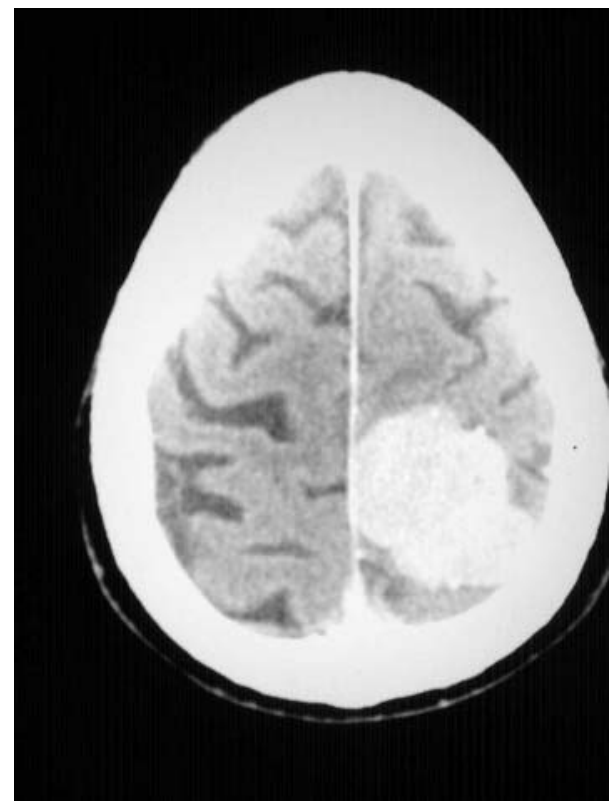

Figure 7 Dorsal stream lesion: CT scan showing left parietal meningioma.

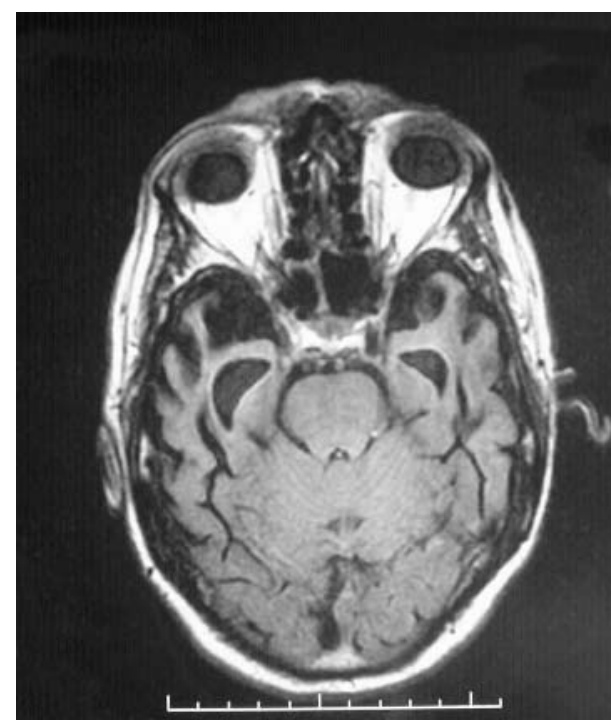

Figure 8 Axial magnetic resonance image (MRI) showing bilateral anterior temporal lobe atrophy in semantic dementia.

system) (table 1). Examples of peripheral alexia include impaired visual acuity as a result of ocular problems, or a visual field defect, even if this does not involve central fixation. Disorders of visual attention such as visual neglect may also impair reading activity. For example, neglect dyslexia results in the patient being unable to read the left hand side of words - for example, for SISTER, the patient will only perceive -TER. On the motor side, impaired ability to coordinate eye movements such as ocular motor apraxia or saccadic intrusions may also impair reading ability.

Alexia without agraphia represents a high level visual deficit resulting in inability to read, and is an example of a disconnection syndrome. Here there is an inability to comprehend written material. The patient can write what he has seen, but is then unable to read back what he has written. It is a sort of category-specific form of visual agnosia for words, in some ways similar to prosopagnosia. The deficit

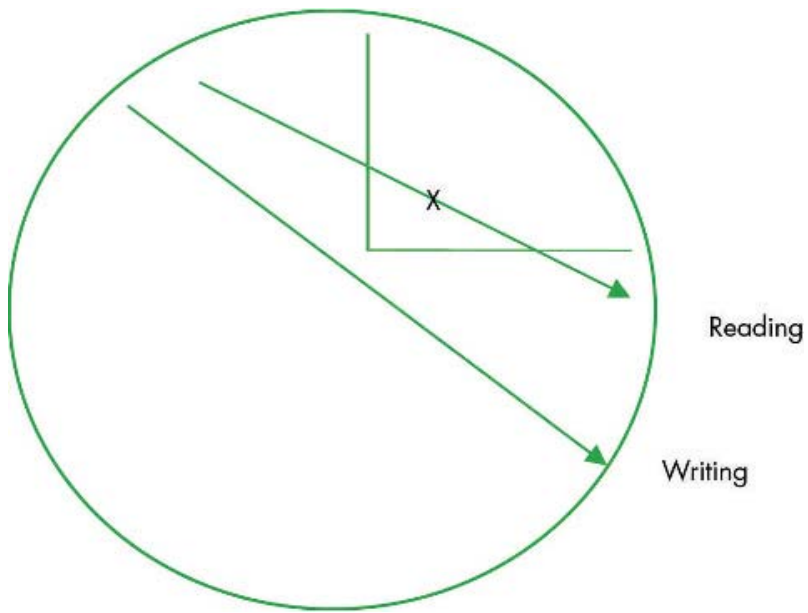

Figure 9 Figure illustrating the disconnection syndrome of alexia without agraphia. Seen written material cannot access Wernicke's area, resulting in alexia. The fibres from the occipital cortex to the motor cortex are, however, spared, so the patient may write what he has seen. 


\begin{tabular}{|c|c|c|}
\hline & & Site of pathology \\
\hline \multicolumn{3}{|l|}{ Peripheral dyslexia } \\
\hline Alexia without agraphia & Disconnection syndrome & Left medial occipital lobe \\
\hline Neglect dyslexia & Difficulty with left side of words or page & Right hemisphere \\
\hline \multicolumn{3}{|l|}{ Central dyslexia } \\
\hline Surface dyslexia & $\begin{array}{l}\text { Loss of semantic knowledge } \\
\text { Difficulty with irregular words }\end{array}$ & Left temporal lobe \\
\hline Deep dyslexia & $\begin{array}{l}\text { Only able to read via meaning, not by letter-by-letter. } \\
\text { Unable to read non-words (e.g. CHOG, LAVE) }\end{array}$ & Left hemisphere \\
\hline
\end{tabular}

is caused by the inability of perceptual information from primary visual cortex to get to language areas-that is, they are disconnected. The patient can recognise words spelled aloud, showing that this is an access problem rather than a primary language deficit. Preserved writing illustrates that pathways from primary visual cortex to pre-motor and motor cortex involved in control of writing movements are preserved. It is best understood by reference to fig 9 .

The syndrome is often accompanied by a right homonymous hemianopia, colour anomia, or achromatopsia, and occurs with lesions affecting the left occipital lobe and the posterior fibres of the corpus callosum. This syndrome is rare and more often overlooked.

\section{Disorders of face perception}

Prosopagnosia refers to a patient's inability to recognise a person simply by studying their face. Once other means of recognition come into play (for example, if the person has a characteristic voice or gait, etc), this allows access to unique semantic identifying information-that is, there is no loss of knowledge of the person. Covert recognition of apparently unrecognised faces is supported by studies of galvanic skin responses, these being greater when viewing faces that were previously known, implying that face identification may occur at unconscious levels.

Prosopagnosia is best understood by means of employing current models of face recognition (fig 10). It can occur with ventral occipitotemporal pathology, especially right sided. ${ }^{4}$

\section{Other face recognition disorders}

Several other conditions previously thought to be of psychiatric origin are now considered organic, with cognitive neuropsychology now providing plausible explanations for their phenomenology. ${ }^{5}$

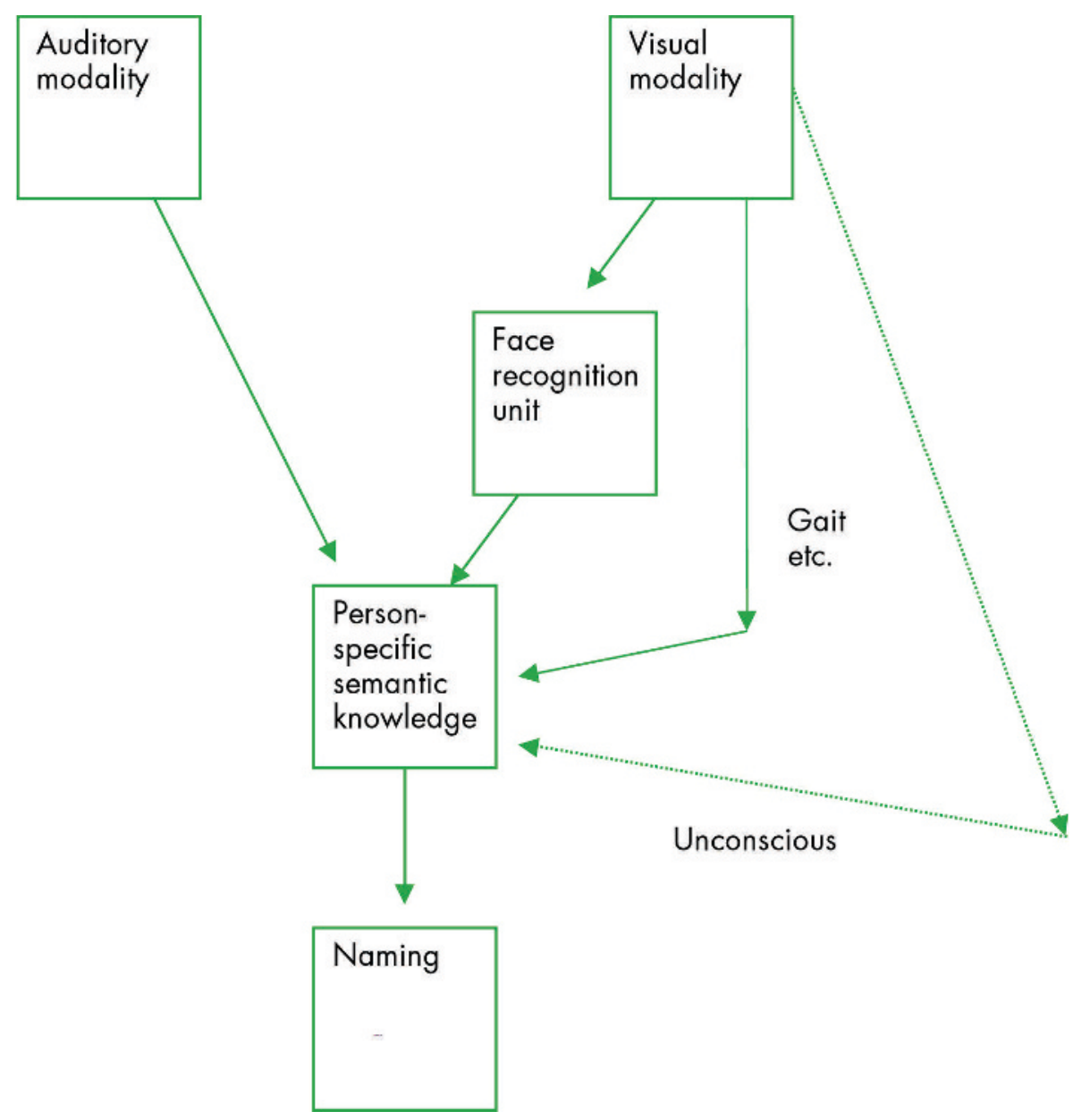

Figure 10 A model of face processing. Prosopagnosia results in damage to face recognition units. 
Capgras syndrome is a form of delusional misidentification in which the patient believes that familiar people have been replaced by impostors. Attempts to explain this condition involve theories of face recognition. Normally, it is claimed that when viewing a face, the conscious stream will result in recognition, while the unconscious stream may invoke feelings of empathy if it is a liked person. Normally, there is no conflict between these parallel streams, and recognition occurs. It is claimed that in Capgras, there may be damage to streams of unconscious processing. This can result in a dissociation between the conscious stream identifying a person as, say, wife, and the lack of unconscious empathic feelings. The lack of concordance between these two streams (that is, looks like wife but no feelings of empathy) may therefore be resolved cognitively by the contention that the loved one has been replaced by an impostor (explaining the dissonance). The lack of concordance between conscious and unconscious streams has been ascribed to a disconnection between the cognitive and emotional streams, anatomically represented by fusiform gyrus of the right temporal lobe and the limbic system, respectively.

Fregoli syndrome is the clinical mirror image of Capgras in that the patient will accuse strangers of being a familiar person in disguise. Intermetamorphosis is a condition where the patient, on looking at a real face, has a subjective experience of the face morphing from one known face into another, and is thought to represent inappropriate activation of face recognition units.

\section{Disorders of colour}

Focal impairments of the colour system mirror deficits to the semantic system in general. Achromatopsia refers to a loss of the ability to perceive colours. Patients usually describe this as like watching black-and-white television. It results from lesions affecting the medial occipito-temporal region, in particular fusiform gyri.

Colour agnosia is not a perceptual deficit (as demonstrated by intact matching tasks) but reflects a loss of semantic knowledge of colour. It is really a category specific semantic memory impairment for colour, and the term "colour agnosia" is unfortunate.

In colour anomia, there is both preserved perception and semantic knowledge regarding colour, but simply a deficit in colour naming.

These deficits may be tested by assessing colour discrimination, colour knowledge, and colour naming.

\section{Deficits of dorsal stream Disorders of motion perception}

Selective impairment of motion perception is rare, and tends to be associated with damage to areas of extrastriate visual cortex analogous to V5-that is, bilateral lesions of the lateral occipitotemporal area. Patients may have no impression of motion in depth or of rapid motion. Fast targets appear to jump rather than move. Particular difficulties are encountered judging the speed and direction of cars.

\section{Disorders of spatial perception}

Balint syndrome is a disorder of spatial perception comprising three aspects: simultanagnosia (the inability to comprehend a complex scene in its entirety-that is, only one component of the scene is perceived at a time), optic ataxia (inability to reach by hand for targets presented visually), and ocular motor apraxia (inability to direct gaze to a visual target). Patients complain of visual difficulties, and may appear functionally blind. They exhibit a curious searching head thrust, by which they aim to search their environment item by item (sometimes unfortunately but accurately compared to the head thrusts of a hen searching for food). Pathology usually is bilateral superior parieto-occipital. It may be cerebrovascular due to "watershed" infarction, or be neurodegenerative as in the posterior cortical atrophy variant of Alzheimer's disease.

Dressing apraxia and constructional "apraxia" Confusingly, these are not apraxias as such, but are rather visuospatial deficits resulting in difficulty dressing and drawing. Dressing apraxia may be tested by asking the patient to don a jacket which has had the sleeves deliberately turned inside out. Bedside tests of constructional apraxia include drawing overlapping pentagons, the Neckar cube, or a clock face. Left hemisphere damage results in simplified drawings, while right sided pathology leads to an "explosion" of the constituent parts of the drawing.

\section{Other higher order deficits of vision Topographagnosia}

Getting lost in familiar surroundings can be due to deficits in either ventral or dorsal visual association cortices.

\section{Anton's syndrome}

This is a form of anosognosia restricted to vision, in which the patient denies there is any visual disturbance despite being functionally blind. It is associated with pathology affecting the primary visual cortex.

\section{Blindsight}

The existence of this condition is disputed, but it is claimed that in the context of blindness caused by primary visual cortex damage, residual unconscious visual function may occur, subserved by subcortical structures such as the lateral geniculate nucleus.

\section{Visual gain}

Pathological gain of visual function can result in positive phenomena-that is, hallucinations. ${ }^{6}$ Visual hallucinations are a strong pointer to organic disease. Common causes include the acute confusional state and Lewy body disease.

\section{Charles Bonnet syndrome}

This comprises positive visual phenomena occurring in areas of visual field deficit, whether this be total or partial. It often arises in the elderly as a result of ocular pathology-for example, age related macular degeneration. The images tend to be complex (for example. animals, people) and insight is usually retained.

\section{Peduncular hallucinosis}

Following midbrain stroke, vivid hallucinations may arise, which tend to occur in the evenings, and have a tendency to disappear over weeks.

\section{HEARING}

Drawing analogies with vision, normal hearing requires intact end organs which feed information to the primary auditory cortex. Again, information is initially strongly tonotopically represented, but loses this as it is processed in the association cortex, which is specialised for types of 
auditory information-for example, speech, music, and environmental sounds.

\section{Hearing loss \\ Auditory agnosias}

The inability to understand the meaning of sounds in the context of preserved basic auditory perception is called auditory agnosia. Strictly speaking, Wernicke's aphasia is a form of auditory agnosia for words, though language disorders are outwith the remit of this review. In practice the term "auditory agnosia" tends to be applied to non-verbal sounds-for example, environmental sounds such as traffic, aircraft noise, etc. Most patients with this have bilateral lesions of the auditory cortex. The disorder is normally of acute onset, and initially the patient becomes almost entirely deaf-that is, cortically deaf. Normally, however, this improves such that the patient hears auditory stimuli. Auditory perception returns to normal (tested clinically by the examiner snapping fingers behind patient's head) but the patient remains unable to identify the sound. Although restricted category specific forms may exist, most auditory agnosias refer to a wide group of environmental sounds.

\section{Hearing gain}

Auditory hallucinations

While hearing voices, especially if threatening or accusatory, is more likely to be due to psychiatric illness, organic disease can also result in auditory hallucinations. These may be musical in nature, and are associated with temporal pathology, often non-dominant. ${ }^{7}$

\section{OTHER SENSORY MODALITIES}

Tactile agnosias have been described - that is, an inability to access semantic knowledge of an item by touch, yet with preserved semantic knowledge of the item when accessed through other modalities such as vision.

Extracampine hallucinations involve the patient sensing a human presence near them, yet they see no one and there is indeed no one there. While this has been reported in normal individuals under severe stress, such as Antarctic explorers, it is well recognised in Lewy body dementia. ${ }^{8}$

\section{THE PARAXIAS}

\section{Motor function}

\section{Normal motor function}

The formulation of an action requires the will and intention to do such a task, and is generated in prefrontal cortex. This signal is then used to activate left fronto-parietal systems that in turn activate motor engrams (for example, the pattern and sequence of movements needed to light a match) in premotor cortex. This is then fed down to the primary motor cortex and by the corticospinal tracts to muscle, with modulation from cerebellum and basal ganglia.

\section{LOSS OF MOTOR FUNCTION \\ Apraxia}

Praxis refers to the ability to perform skilled movements. Dyspraxia refers to difficulty performing motor acts which cannot be accountable by lack of understanding of the task, weakness, or proprioceptive loss. (Apraxia can even occur in the presence of an additional deficit such as hemiparesis, but to qualify as apraxia, the examiner must be confident that the apparent difficulty performing the motor act cannot be explained by the hemiparesis alone.) Many patients with

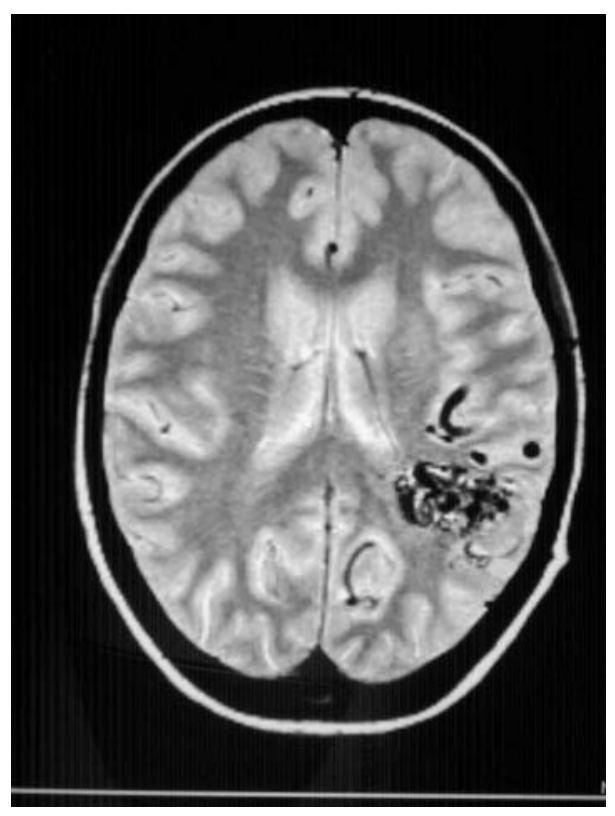

Figure 11 MRI showing left parietal arterio-venous malformation.

dyspraxia are unaware of their deficits, thus the examiner should specifically enquire from the patient and caregiver as to how the patient manages activities of daily living such as brushing teeth, combing hair, using kitchen utensils, etc. Dyspraxia is frequently missed by clinicians, and may then be detected by occupational therapists or physiotherapists-for example, when seeing patients recovering from stroke.

\section{Anatomy and pathology of apraxia}

Left parietal or frontal premotor lesions are most commonly associated with dyspraxia (fig 11). It is thought that it results from loss of motor engrams or a disconnection between praxis systems. Orobuccal apraxia occurs with insular and left inferior frontal lesions.

The most common causes of apraxia are stroke and neurodegenerative disease such as Alzheimer's or corticobasal degeneration-progressive isolated limb apraxia strongly suggesting the latter. ${ }^{9}$

\section{Subdivisions of apraxia}

Confusingly, traditional subdivisions of apraxia, particularly ideomotor and ideational, are used in an inconsistent manner. Clinically it is often sufficient simply to describe which body parts are affected (for example, limbs or orobuccal), and to describe which movements are impaired. If a more detailed knowledge of praxis is required, then the following cognitive neuropsychological model of praxis and its deficits may be helpful.

Praxis is conceptually difficult, and it may help for the reader to draw analogies between the following account of praxis, and the more widely understood language system. Praxis requires both a conceptual system of knowledge of tool functions and actions (for example, the purpose of a screwdriver), and a production system including sensorimotor action programmes concerned with the generation and control of movement (that is, the ability to move a limb through space in the correct direction and with appropriate speed). ${ }^{10}$ 


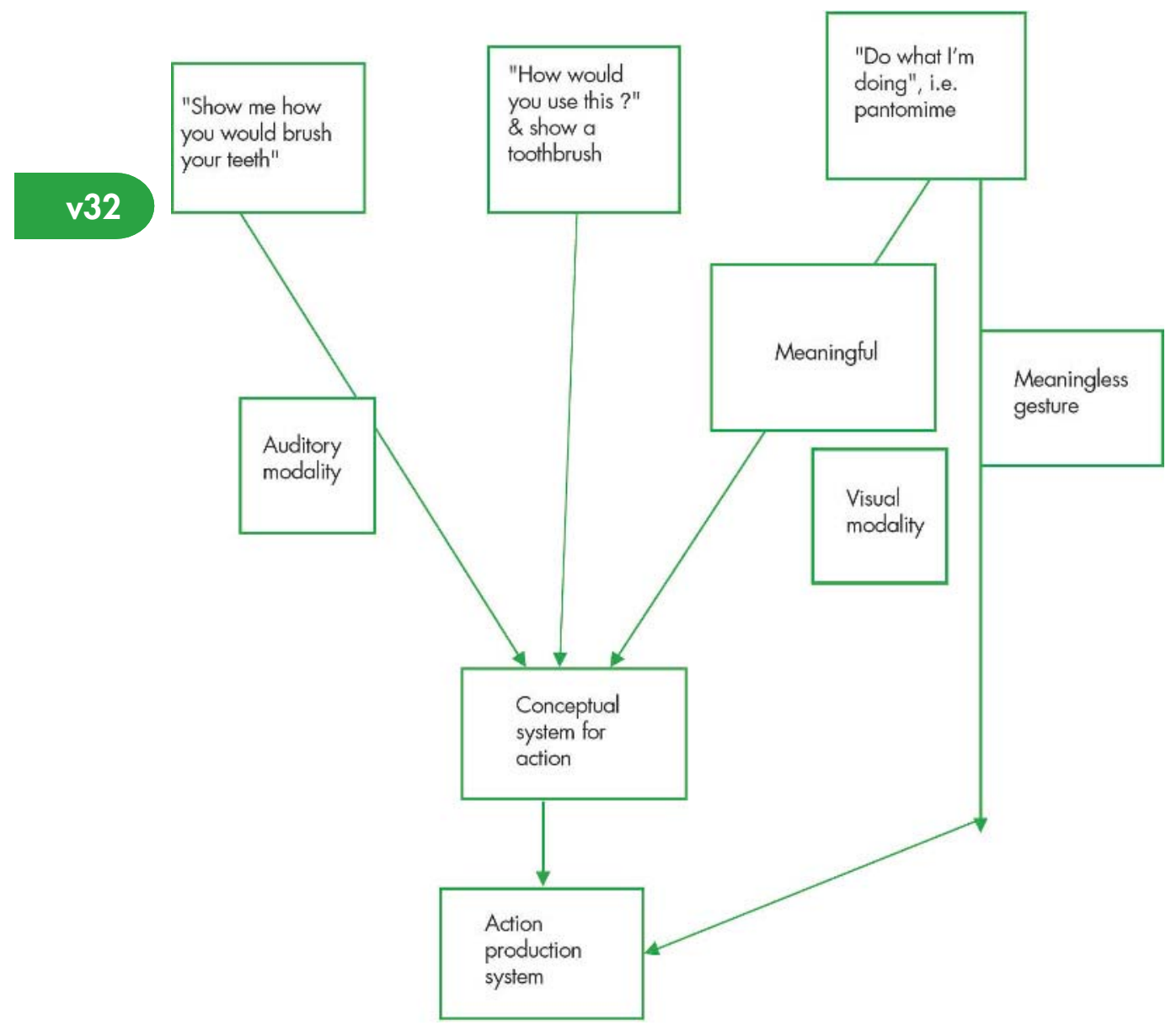

Figure 12 A model of praxis, which can be accessed through various sensory modalities.

\section{How to examine for dyspraxia}

It is impossible to draw any meaningful conclusions about the presence or absence of praxis, unless tests for it are interpreted in the context of the rest of the neurological examination-hence a full neurological examination is essential.

Given the above confusion regarding subdivisions of praxis, there is no clear consensus as to how best to test praxis. If, however, we employ the above classification of the conceptual system for action (that is, knowledge of actions and tools) and the action production system (that is, the ability to carry out motor programmes), then this lends itself to a logical means of exploring dyspraxia in patients. The following account of testing praxis is exhaustive and lends itself more to clinical research than clinical practice. It is best read in conjunction with fig 12 .

Graham et $\mathrm{l}^{10}$ assessed the conceptual system for action by testing naming of actions, naming of tools, specification of tool use (for example, "What would you use a toothbrush for ?", and action recognition ("Which is the correct movement for brushing teeth?" with the examiner miming both the target action and distractors). The action production system was assessed by hand and finger position imitation, execution of familiar action sequences (for example, "Fold a piece of paper, place in envelope and seal the envelope"), and execution of single familiar actions. Execution of single familiar actions can be further assessed by verbal command "Show me how you would use a toothbrush", imitation of examiner's mime, ability to pantomime usage while looking at the tool "Pretend you have this in your hand. Show me how you would use it", and by actual tool use "Take this in your hand and show me how you would use it".

A simpler means of testing praxis at the bedside is to ask the patient to imitate gestures, or to gesture to command. Use of imagined objects should also be tested. The above should be done both for limb movements and for orobuccal movements. Finally, a sequencing task such as the Luria three-step test or alternating hand movements can be done.

Table 2 illustrates a useful series of commands which allow praxis to be tested at the bedside. ${ }^{11}$

\section{Ideational apraxia}

Damage to the conceptual system leads to both impaired gesture comprehension and discrimination, as well as a production deficit. Patients have difficulty performing in response to command and imitation and do not discriminate well between poorly and well performed acts. Patients may make content and tool selection errors, and exhibit loss of tool-object action knowledge (for example, using a screwdriver as a hammer). Preserved tool naming shows that this deficit cannot be explained on the basis of an object agnosia. Patients may also show a loss of tool-object association knowledge (for example, when shown a partly driven nail, they may choose a screwdriver rather than a hammer). This is best termed an ideational apraxia (although others use the 
Table 2 Schema for examining praxis: "Show me how you would ..." If defective by command, test by imitation.

\begin{tabular}{ll}
\hline Buccofacial & $\begin{array}{l}\text { Blow out a match } \\
\text { Lick lips } \\
\end{array}$ \\
& Cough \\
Sip through a straw \\
Limb & Gestures
\end{tabular}

Wave goodbye Beckon "come here"

Salute, like a soldier

Hitch a lift

Object use

Comb hair

Brush teeth

Use scissors

Hammer a nail

term conceptual apraxia or even posterior ideomotor apraxia, and reserve the term ideational for another disorder). It is most commonly seen in Alzheimer's disease

\section{Ideomotor apraxia}

By contrast, ideomotor dyspraxia relates to an impaired action production system. Although it also results in production deficits whether by command or imitation, it can be discriminated from ideational dyspraxia in that gesture comprehension and discrimination are preserved.

Both spatial and temporal production errors may occur in ideomotor apraxia. The former include postural errors-that is, use of body part as tool (for example, using finger as toothbrush; this can occur in normal controls, but if it persists when the patient has been told not to, it is pathological). Deficits in spatial orientation (for example, keeping scissors in the sagittal plane) and spatial movement (for example, rotating at the shoulder rather than pronating/ supinating when using a screwdriver) may also occur. Timing errors relate to the fluidity of movement (for example, cutting bread with a knife should employ regular sinusoidal movements). Lesions affecting the premotor cortex may cause this type of dyspraxia.

Some patients, often with bilateral frontal and parietal dysfunction as in dementia, are unable to carry out a series of acts in the proper sequence (for example, folding paper, putting in envelope, sealing the envelope). Confusingly, this has been termed ideational apraxia, but it is really a failure of execution of familiar action sequences, and hence a measure

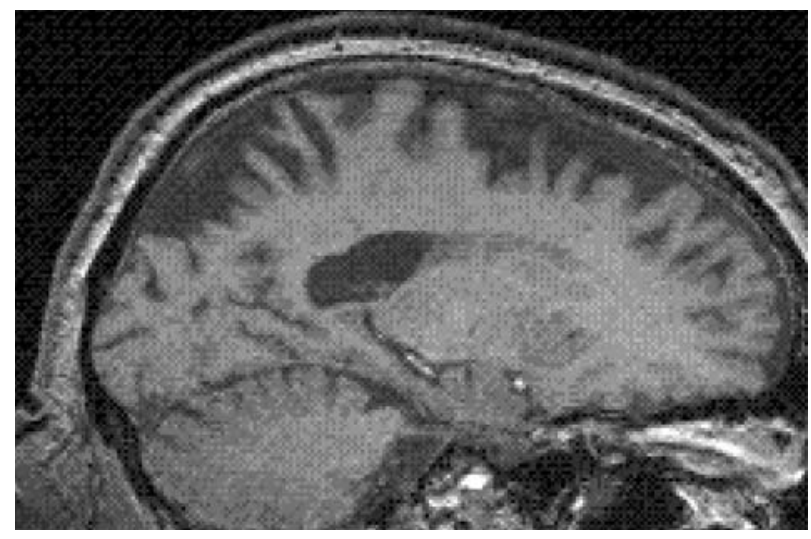

Figure 13 Sagittal MRI of corticobasal degeneration illustrating striking frontoparietal atrophy, but with sparing of primary sensorimotor cortex.

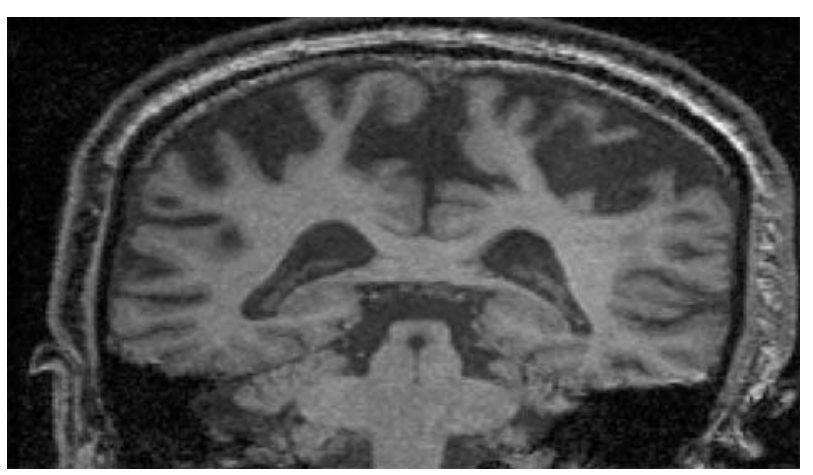

Figure 14 Coronal MRI of corticobasal degeneration showing striking gyral atrophy.

of the integrity of the action production system, and is therefore a type of ideomotor apraxia.

\section{Conduction apraxia}

This results in greater impairment imitating movements than when pantomiming to verbal command, and is analogous to conduction aphasia. The location of the lesion responsible is unknown.

\section{Verbal disassociation apraxia}

Here, the patient cannot gesture normally to command but can perform well both when imitating the examiner, and when using actual tools and objects. This suggests that both the conceptual system for action and action production system are preserved, but simply cannot be accessed by verbal command.

\section{Callosal apraxia}

The left frontal and parietal areas are dominant for higher order motor function. A lesion affecting the anterior corpus callosum will not impair the left hemisphere's ability to control movement of the right arm and leg. The callosal lesion will, however, impair the left hemisphere's ability to control the right premotor cortex, and can therefore result in left sided apraxia caused by this disconnection.

\section{Gain of motor function}

"Toxic gain" of motor function may occur at prefrontal, premotor, and motor levels (an example of the latter being focal motor seizures).

The prefrontal cortex may exert an inhibitory effect on the ability of perceptual input to result in motor response-that is, it lets us carve an autonomous path in the world rather than be a slave to our environment. Prefrontal pathology can remove this inhibition, leading to a compulsion to act on the surroundings (that is, environment dependency syndrome or forced utilisation behaviour). A patient with this condition, on being taken into a room with hammer, nails, and a picture, will hammer a nail into the wall and hang the picture. If asked why he did that, he will say "I assume from the items being there that this is what you wanted me to $\mathrm{do}^{\prime \prime} .^{12} 13$

\section{Alien hand syndrome}

Often associated with apraxia, in this disorder the limb may perform movements not under volitional control, as if it has a will of its own. Severe alien hand on one side may result in intermanual rivalry, such as the anarchic hand trying to 


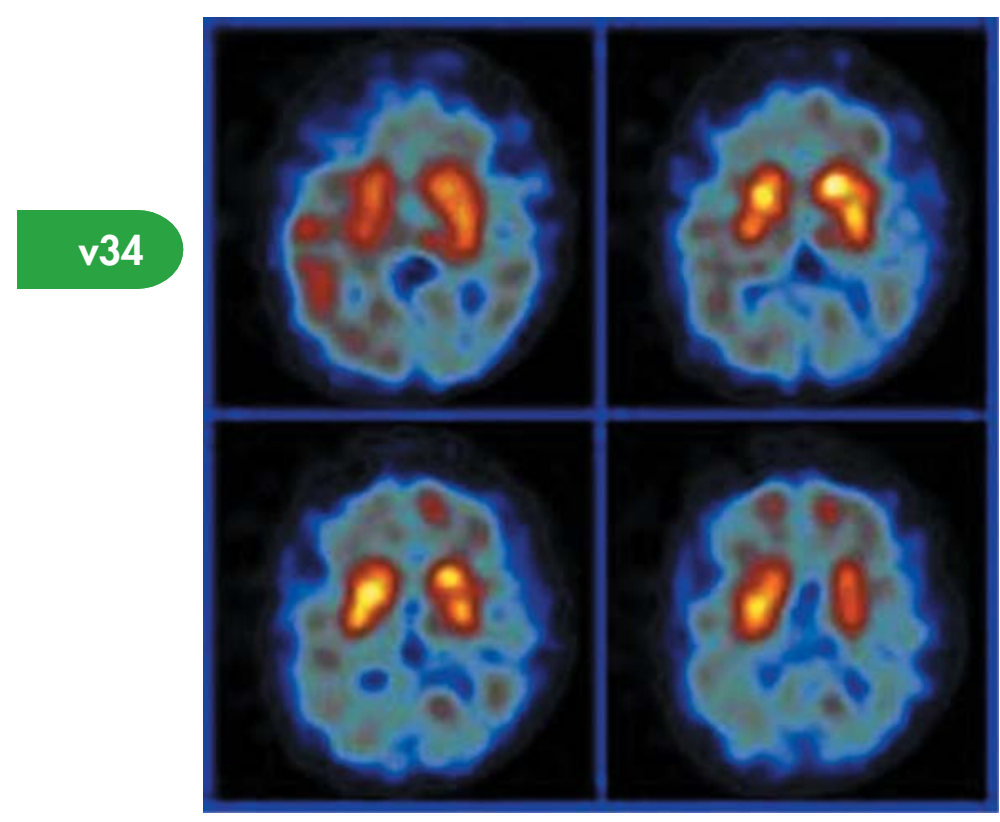

Figure 15 D2-single photon emission computed tomography (SPECT) scan illustrating reduced post-synaptic dopamine receptors as occurs in Parkinson-plus syndromes, as in corticobasal degeneration here.

throttle the patient while the other hand under volitional control tries to fend it off, but such circumstances are rare and tend to occur transiently in the context of acute cerebrovascular disease. Progressive isolated alien limb is essentially pathognomonic of corticobasal degeneration (figs 13-15).

\section{CONCLUSION}

To summarise, while memory and language disorders tend to dominate cognitive neurology, it is important to have at least a working knowledge of other cognitive deficits

- Disorders of gnosis and praxis may result in either pathological loss or pathological gain in function.

- Agnosias may involve any sensory modality, not just vision.
- Deficits of visual processing are best divided into disorders of the ventral "what" stream and of the dorsal "where" stream.

- Agnosias can be differentiated from more central semantic deficits by assessing the same object using different modalities. If knowledge can be accessed via other modalities, then there is a modality specific agnosia rather than a multi-modal semantic deficit.

- Apraxia is often missed in the acute stage, as it can be masked by coincident hemiparesis. It is often first noticed by physiotherapists in stroke rehabilitation.

- Subdivisions of apraxia are confusing. Perhaps the most logical subdivision is into disorders of either the conceptual system for action (that is, semantic knowledge of tools and actions), or the action production system (that is, the actual motor programmes needed to carry out tasks).

- Progressive isolated alien limb is very suggestive of corticobasal degeneration.

\section{REFERENCES}

1 Rees G, Kreiman G, Koch C. Neural correlates of consciousness in humans. Nature 2002;3:261-70.

2 Zeman A. Consciousness. Brain 2001;124:1263-89.

3 Ungerleider L, Mishkin M. Two cortical visual systems. In: Ingle DJ, Mansfield RJW, Goodale MS, eds. The analysis of visual behaviour. Cambridge, Massachusetts: MIT Press, 1982:549-86.

4 Evans JJ, Heggs AJ, Antoun N, et al. Progressive prosopagnosia associated with selective right temporal lobe atrophy. Brain 1995;1 18:1-13.

5 Ellis HD, Young AW. Accounting for delusional misidentifications. Br J Psychiatry 1990;157:239-48.

6 Ffytche D. Visual hallucination and illusion disorders: a clinical guide. Advances in Clinical Neuroscience and Rehabilitation 2004;4(2):16-18.

7 Berrios GE. Musical hallucinations. Br J Psych 2005; 156:188-94.

8 Chan D, Rossor MN. "-but who is that on the other side of you?" Extracampine hallucinations revisited. Lancet 2002;360:2064-6.

9 Spatt J, Bak T, Bozeat S, et al. Apraxia, mechanical problem solving and semantic knowledge: contributions to object usage in corticobasal degeneration. J Neurol 2002;249:601-8.

10 Graham NL, Zeman A, Young AW, et al. Dyspraxia in a patient with corticobasal degeneration: the role of visual and tactile inputs to action. J Neurol Neurosurg Psychiatry 1999;67:334-44.

11 Hodges JR. Cognitive assessment for clinicians. Oxford: Oxford University Press, 1994.

12 Lhermitte $F$. Utilisation behaviour and its relation to lesions of the frontal lobes. Brain 1983; 106:237.

13 Lhermitte F. Human autonomy and the frontal lobes. II: Patient behaviour in complex and social situations-the "environmental dependency syndrome", Ann Neurol 1986; 19:335. 